\title{
Effect of partial saturation on the stability of shallow foundations above the water table
}

\author{
Brunella Balzano, Alessia Amabile,Gianfranco Urciuoli, and Alessandro \\ Tarantino,
}

\begin{abstract}
Granular 'cohesionless' soils above the water table are partially saturated but are commonly assumed to be dry in geotechnical practice. Accordingly, 'drained' shear strength is calculated by replacing the 'saturated' effective stress with the total stress. The 'dry soil' assumption neglects the effect $t$ of suction on shear strength and, as a result, geo-structures are over designed. To investigate the implications of this assumption, this paper presents an approach to calculate the bearing capacity of shallow foundations above the water table taking into account the effects of partial saturation. This approach is based on the upper bound theorem of plasticity. The bearing capacity of a strip foundation in granular soils is calculated and the solution obtained by taking into account the effects of partial saturation is compared with the solution obtained from the classical 'dry' approach.
\end{abstract}

Keywords: partial saturation, shallow foundation, ultimate limit state

\section{Introduction}

Non-clayey 'cohesionless' soils above the water table are generally assumed to be dry in routine engineering calculations. Nonetheless this is rarely the case. Soils above the water table are partially saturated and have shown to exhibit significant-

Brunella Balzano

Università di Napoli Federico II, Napoli, Italy, e-mail: fonbal@hotmail.com Alessia Amabile

Università di Napoli Federico II, Napoli, Italy, e-mail: alessia_ama@hotmail.it Ginfranco Urciuoli

Università di Napoli Federico II, Napoli, Italy, e-mail: gianurci@unina.it

Alessandro Tarantino

University of Strahclyde, Glasgow, UK, e-mail: alessandro.tarantino@strath.ac.uk 
ly higher shear strength than dry soils. Practitioners and academicians find it convenient to disregard the contribution of partial saturation to shear strength as this leads to conservative design. However, significant costs might be saved if new geo-structures are designed to account for the effects of partial saturation. In addition, geotechnical engineers are often confronted with existing hazardous geostructures, e.g. unstable slopes or foundations. In this case, a realistic analysis of the current state of stress, including the characterisation of the partially saturated zone above the water table, is essential to assess the causes of instability and, hence, to design appropriate remedial measures.

To quantify the effects of partial saturation on the stability of geostructures, methods should be developed to analyse collapse conditions in partially saturated soils. This paper presents an approach based on the upper bound theorem of plasticity to calculate the bearing capacity of shallow foundations. For the sake of simplicity, a simple two-block mechanism is considered and the case of 'cohesionless' granular materials is analysed. The accuracy of the two-block mechanism is first examined by considering the case of dry/saturated soil. Afterwards, the solution for partially saturated soils above the water table is derived and compared with the solution obtained by assuming the soil to be dry above the water table.

\section{Upper bound theorem of plastic collapse}

The upper and lower bound theorems of plastic collapse set limits to the collapse load of a structure and can be proved for the case of perfectly plastic materials. In the present paper the upper bound has been considered to calculate the bearing capacity of a foundation under partial saturation conditions.

To apply the upper bound theorem, firstly a kinematically admissible mechanism needs to be considered and secondly the external work $W_{\mathrm{e}}$ and the internal energy dissipation $W_{\mathrm{i}}$ need to be equated.

The external work is given by:

$$
W_{e}=\sum \vec{F} \cdot \vec{\delta}
$$

where $\overrightarrow{\mathrm{F}}$ are the external forces and $\vec{\delta}$ are the displacements of the application points of the forces.

The internal work is given by:

$$
W_{i}=\int_{V}(\sigma \cdot \varepsilon+\tau \cdot \gamma) d V
$$

where $\sigma$ and $\tau$ are the normal total stress and the tangential stress respectively, $\varepsilon$ and $\gamma$ are the normal and the shear strain respectively, and $V$ is the volume of the shear band. For non-clayey 'cohesionless' geomaterials, the shear strength criterion can be written as follows (Tarantino \& El Mountassir, 2012): 


$$
\tau=c^{\prime}+\left(\sigma-u_{w} S_{r}\right) \tan \phi^{\prime}
$$

where $c^{\prime}$ is the effective cohesion, $u_{\mathrm{w}}$ is the pore-water pressure, $S_{\mathrm{r}}$ is the degree of saturation, and $\phi '$ is the effective angle of shearing resistance.

Assuming a cohesion $c^{\prime}=0$ and developing the integral for $W_{\mathrm{i}}$, the following expression is obtained for the case of planar slip surface:

$$
W_{i}=-\delta_{t} \tan \varphi^{\prime} \int_{L} u_{w} S_{r} d L
$$

where $\delta_{t}$ is the component of displacement tangential to the slip surface having length $L$.

\section{Application of upper bound theorem to saturated/dry soils}

The upper bound theorem of limit analysis was first applied to the calculation of the bearing capacity of shallow foundation involving saturated or dry soils. For the sake of simplicity, the kinematically admissible mechanism considered here consists of only two blocks with planar slip surfaces as shown in Figure 1.
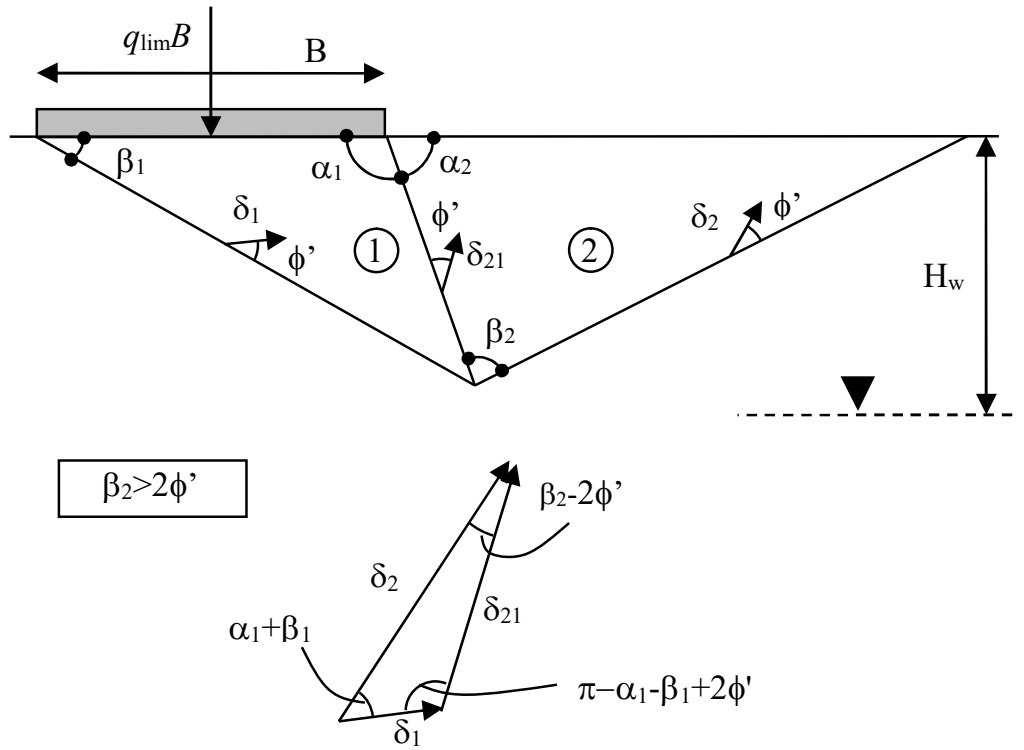

Figure 1. Kinematic mechanism of two blocks and displacement odograph.

In this case, $W_{\mathrm{e}}$ and $W_{\mathrm{i}}$ are expressed as follows: 


$$
\begin{gathered}
W_{e}=\left(q_{l i m} B\right) \delta_{1 v}-\left(q_{0} l_{2}\right) \delta_{2 v}+\gamma S_{1} \delta_{1 v}+\gamma S_{2} \delta_{2 v} \\
\mathrm{~W}_{\mathrm{i}}=-\sin \varphi^{\prime}\left(\mathrm{U}_{1} \delta_{1}+\mathrm{U}_{2} \delta_{2}+\mathrm{U}_{1,2} \delta_{1,2}\right)
\end{gathered}
$$

where $q_{\mathrm{lim}}$ is the bearing capacity of the foundation, $B$ is the width of the foundation, $q_{0}$ is the surcharge pressure, $\delta_{1 \mathrm{v}}$ and $\delta_{2 \mathrm{v}}$ are the vertical components of displacements $\delta_{1}$ and $\delta_{2}, \mathrm{~S}_{1}$ and $\mathrm{S}_{2}$ are the cross-sectional areas of the two blocks, $\gamma$ is the unit weight of the soil, and $U_{1}, U_{2}, U_{1,2}$ are the water thrusts acting on $d_{1}, d_{2}$ and $l_{1}$ respectively (see Figure 1 ). The latter are generally expressed as:

$$
U=\int_{L} u_{w} S_{r} d L
$$

where $S_{\mathrm{r}}=1$ under saturated conditions. If hydrostatic conditions are assumed, $u_{\mathrm{w}}$ is given by:

$$
u_{w}=\gamma_{w}\left(z-H_{w}\right)
$$

where $H_{\mathrm{w}}$ is water table depth and $\gamma_{\mathrm{w}}$ is the unit weight of water. By equating $W_{\mathrm{e}}=W_{\mathrm{i}}$ the bearing capacity $q_{\mathrm{lim}}$ can be expressed as follows:

$$
q_{l i m}=N_{q} q_{0}+N_{\gamma} \gamma \frac{B}{2}+N_{\gamma_{w}} \gamma_{w} \frac{B}{2}+N_{H_{w}} \gamma_{w} H_{w}
$$

The bearing capacity factors $N_{\gamma}, N_{\mathrm{q}}, N_{\gamma \mathrm{w}}$, and $N_{\mathrm{Hw}}$ are functions of the angles $\alpha_{1}$, $\beta_{1}, \beta_{2}$, and $\varphi^{\prime}$ (see Figure 1). $N_{\gamma}$ is the factor associated with the soil unit weight under 'dry' conditions $\left(q_{0}=0, \gamma_{\mathrm{w}}=0, H_{\mathrm{w}}=0\right), N_{\mathrm{q}}$ is the factor associated with the surcharge pressure $\left(\gamma=0, \gamma_{\mathrm{w}}=0, H_{\mathrm{w}}=0\right)$, and $N_{\gamma_{\mathrm{w}}}$ and $N_{\mathrm{Hw}}$ are the factors that account for the pore-water pressure $u_{\mathrm{w}}$ and the position of the water table $H_{\mathrm{w}}$ respectively. The bearing capacity factors for the two-block mechanism are given by:

$$
\begin{gathered}
N_{\gamma}=-\left(\frac{\sin \alpha_{1}+\sin \beta_{1}}{\sin \left(\alpha_{1}+\beta_{1}\right)}+\frac{\sin \alpha_{1}\left(\sin \beta_{1}\right)^{2}\left(\sin \beta_{2}\right)^{2} \sin \left(\alpha_{1}+\beta_{2}-2 \varphi^{\prime}\right) \sin \left(\beta_{2}-\alpha_{1}-\varphi^{\prime}\right)}{\left(\sin \left(\alpha_{1}+\beta_{1}\right)\right)^{2} \sin \left(\beta_{2}-2 \varphi^{\prime}\right) \sin \left(\beta_{1}-\varphi^{\prime}\right) \sin \left(\alpha_{1}-\beta_{2}\right)}\right) \\
N_{q}=\frac{\sin \beta_{1} \sin \beta_{2} \sin \left(\alpha_{1}+\beta_{1}-2 \varphi^{\prime}\right) \sin \left(\beta_{2}-\alpha_{1}-\varphi^{\prime}\right)}{\sin \left(\alpha_{1}+\beta_{1}\right) \sin \left(\alpha_{1}-\beta_{2}\right) \sin \left(\beta_{2}-2 \varphi^{\prime}\right) \sin \left(\beta_{1}-\varphi^{\prime}\right)} \\
N_{\gamma_{W}}=-\frac{\sin \varphi^{\prime} \sin \alpha_{1} \sin \beta_{1}}{\sin \left(\beta_{1}-\varphi^{\prime}\right)\left(\sin \left(\alpha_{1}+\beta_{1}\right)\right)^{2}}\left(\sin \alpha_{1}+\frac{\sin \alpha_{1} \sin \beta_{1} \sin \left(\alpha_{1}+\beta_{1}-2 \varphi^{\prime}\right)}{\sin \left(\alpha_{1}-\beta_{2}\right) \sin \left(\beta_{2}-2 \varphi^{\prime}\right)}+\frac{\sin \beta_{1} \sin \left(\alpha_{1}+\beta_{1}-\beta_{2}\right)}{\sin \left(\beta_{2}-2 \varphi^{\prime}\right)}\right) \\
N_{H_{W}}=\frac{\sin \varphi^{\prime}}{\sin \left(\beta_{1}-\varphi^{\prime}\right) \sin \left(\alpha_{1}+\beta_{1}\right)}\left(\sin \alpha_{1}+\frac{\sin \alpha_{1} \sin \beta_{1} \sin \left(\alpha_{1}+\beta_{1}-2 \varphi^{\prime}\right)}{\sin \left(\alpha_{1}-\beta_{2}\right) \sin \left(\beta_{2}-2 \varphi^{\prime}\right)}+\frac{\sin \beta_{1} \sin \left(\alpha_{1}+\beta_{1}-\beta_{2}\right)}{\sin \left(\beta_{2}-2 \varphi^{\prime}\right)}\right)
\end{gathered}
$$

It can be easily verified that $N_{\gamma}=-N_{\gamma_{\mathrm{w}}}$. This confirms that bearing capacity under saturated conditions can be derived from the 'dry soil' solution by replacing the unit weight $\gamma$ with the effective unit weight $\gamma$ '. 


\section{Comparison of the two-block solution with traditional models for dry/saturated soils}

The expressions traditionally considered for the factors $\mathrm{N}_{\gamma}$ and $\mathrm{N}_{\mathrm{q}}$ are the ones given by Vesic (1975) and the EC7. Vesic and EC7 consider the same expression for $N_{\mathrm{q}}$ :

$$
N_{q}=k_{p} e^{\pi \tan \varphi}
$$

where $k_{\mathrm{p}}$ is the passive earth coefficient. The equations given for $\mathrm{N}_{\gamma}$ by Vesic (1975) and the EC7 respectively are slightly different:

$$
\begin{gathered}
N_{\gamma}=2\left(N_{q}+1\right) \tan \varphi^{\prime} \quad(\text { Vesic, 1975) } \\
N_{\gamma}=2\left(N_{q}-1\right) \tan \varphi^{\prime} \quad(\text { EC7 })
\end{gathered}
$$

The coefficients derived for $N_{\mathrm{q}}$ and $\mathrm{N}_{\gamma}$ by considering the two-block mechanism (see Eq. (10) and (11)) are compared with the EC7 and Vesic's solutions in Figure 2. For the case of the two-block mechanism, the minimum upper bound values for $N_{\mathrm{q}}$ and $\mathrm{N}_{\gamma}$ were derived by minimisation with respect to $\alpha_{1}, \beta_{1}$, and $\beta_{2}$.
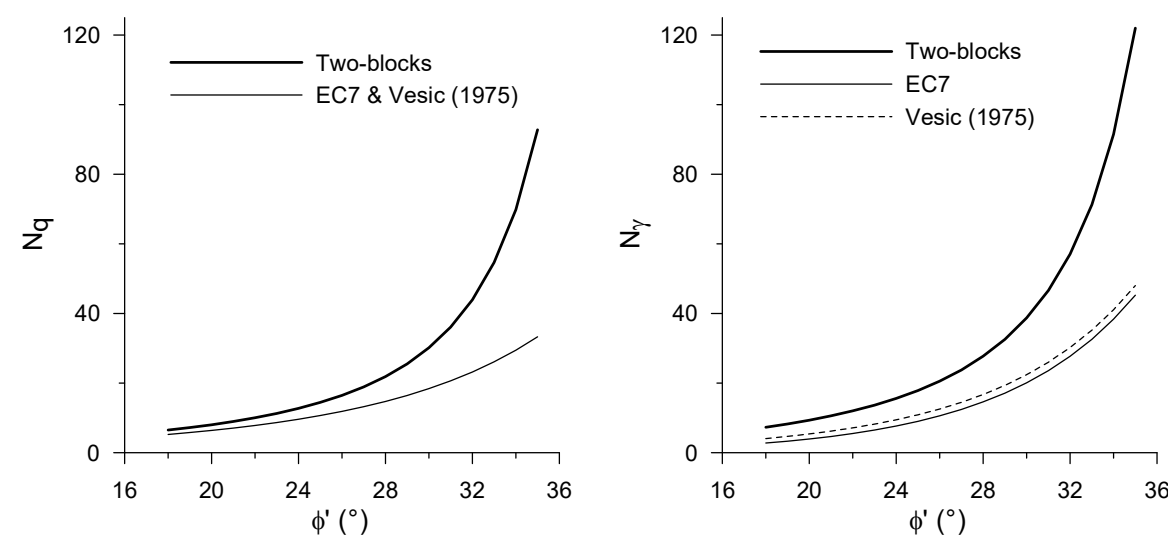

Figure 2. Comparison of factors $\mathrm{N}_{\gamma}, \mathrm{N}_{\mathrm{q}}$ derived from the two-block mechanism with the values provided by Vesic (1975) and EC7.

As shown in the graphs, the two-block mechanism shows an acceptable accuracy at low values of the effective angle of shearing resistance $\varphi$ '. As the angle of shearing resistance increases, the two-block mechanism significantly overestimates the bearing capacity factors. This suggests that the failure mechanism is too simplistic for estimating the bearing capacity of shallow foundations. In this paper, the two-block mechanism will therefore only be used in comparative fashion 
to demonstrate the difference between the 'dry soil' and the 'partially saturated soil' assumptions.

\section{Extension to unsaturated soils}

In unsaturated soils void spaces are partially filled with water and partially filled with gas and the degree of saturation ranges between 0 and 1 . The degree of saturation $S_{\mathrm{r}}$ is related to the pore-water pressure $u_{\mathrm{w}}$ via the water retention function. The function proposed by van Genuchten (1980) is adopted here:

$$
S_{r}= \begin{cases}\left(1+\left(-\alpha u_{w}\right)^{n}\right)^{-m} & \left(u_{w} \leq 0\right) \\ 1 & \left(u_{w}>0\right)\end{cases}
$$

where $\alpha, n$, and $m$ are soil parameters.

By using Eqs. (7), (8), and (17), the water thrusts $U_{1}, U_{2}$ and $U_{1,2}$ can be calculated and the bearing capacity $q_{\lim }$ can be derived by equating the external and internal works given by Eqs. (5) and (6) respectively. It should be noted that the integral in Eq. (7) cannot be solved analytically when using the van Genuchten function and a numerical solution was therefore obtained.

Two different soils were considered in this exercise to quantify the effect of partial saturation on the bearing capacity of shallow foundations, a natural pyroclastic silty sand (Nicotera et al. 2010 and Papa et al. 2008) and a reconstituted silt (Geiser et al. 2006). For both soils, the shear strength criterion in Eq. (3) holds as shown by Tarantino and El Mountassir (2012). The water retention and shear strength parameters for the two soils are reported in Table 1.

Table 1. Soil parameters

\begin{tabular}{llllll}
\hline & $\gamma\left[\mathbf{k N} / \mathbf{m}^{3}\right]$ & $\begin{array}{l}\boldsymbol{\varphi}^{\prime} \\
{\left[{ }^{\circ}\right]}\end{array}$ & $\begin{array}{l}\boldsymbol{\alpha} \\
{\left[\mathbf{k P a}^{-1}\right]}\end{array}$ & $\boldsymbol{n}$ & $\boldsymbol{m}$ \\
\hline Silty sand (Nicotera et al. 2010) & 15 & 36.9 & 0.065 & 1.67 & 0.400 \\
Silt (Geiser et al. 2006) & 18 & 30 & 0.022 & 4.05 & 0.177 \\
\hline
\end{tabular}

A water table depth $H_{\mathrm{w}}=4 \mathrm{~m}$ and a foundation width $B=1 \mathrm{~m}$ were considered. As a result, the failure mechanism entirely develops above the water table. The comparison between the bearing capacity in dry soil (as assumed in routine geotechnical design) and in partially saturated soil is presented in Table 2 . The bearing capacity derived under the realistic assumption of partially saturated soil is significantly higher than the value obtained by assuming the soil dry $(60 \%$ and $98 \%$ for the silty sand and silt respectively). 
Table 2. Comparison of bearing capacity $q_{\text {lim }}$ obtained from 2 blocks model

\begin{tabular}{llll}
\hline & $\mathrm{q} \lim (\mathrm{kPa})$ & $\mathrm{q} \lim (\mathrm{kPa})$ & $\frac{\mathrm{q}_{\text {unsat }}-\mathrm{q}_{\text {dry }}}{\mathrm{q}_{\text {dry }}}$ \\
\hline & $\mathrm{dry}$ & unsaturated & \\
\hline Silty sand (Nicotera et al. 2010) & 5698 & 9112 & $60 \%$ \\
Silt (Geiser et al. 2006) & 952 & 1881 & $98 \%$ \\
\hline
\end{tabular}

\section{Conclusions}

The paper has investigated the effect of partial saturation on the bearing capacity of shallow foundations. In particular, the bearing capacity derived by assuming the soil partially saturated has been compared with the bearing capacity derived by assuming the soil above the water table to be dry, as is generally the case in routing geotechnical design.

The bearing capacity was calculated using the upper bound theorem of plasticity and considering a two-block mechanism. The two-block mechanism significantly overestimates the bearing capacity at relatively high angles of shearing resistance. In this paper, this simplistic mechanism was only used in a comparative fashion to investigate the difference between the 'dry soil' and the 'partially saturated soil' assumptions.

Preliminary results show that the effect of partial saturation is significant and this encourages further experimental and theoretical research on the collapse behaviour of geotechnical structures above the water table.

\section{References}

Eurocode 7: Geotechnical design - Part 1: General rules, BS EN 1997-1:2004

Geiser F., Laloui L., Vulliet L. (2006). Elasto-plasticity of unsaturated soils: laboratory test results on a remoulded silt. Soils and Foundations Journal, 46(5): 545-556.

Nicotera M.V., Papa, R. and\& Urciuoli G. (2010). "An experimental technique for determining the hydraulic properties of unsaturated pyroclastic soils". Geotechnical Testing Journal, Vol. 33, No. 4. DOI: $10.1520 /$ GTJ102769.

Papa R., Urciuoli G., Evangelista A. and Nicotera M.V. (2008). Mechanical properties of unsaturated pyroclastic soils affected by fast landslide phenomena. In Unsaturated Soils, Advances in Geo-Engineering, Proceedings of the 1st European Conference, E-UNSAT 2008, Durham, United Kingdom, D.G. Toll, C.E. Augarde, D. Gallipoli, and S.J. Wheeler (eds), Taylor \& Francis, pages 917-923.

Tarantino A \& El Mountassir G (2012). Cheap unsaturated soil mechanics. Submitted to Engineering Geology.

van Genuchten, M. Th. 1980. A closed-form equation for pre- dicting the hydraulic conductivity of unsaturated soils. Soil. Sci. Soc. Am. J. 44:892-898

Vesic, A. S. (1975). Bearing capacity of shallow foundations. In Foundation Engineering Handbook, Winterkorn, H.F. and Fang, H.Y. eds., Van Nostrand, New York, 121-147 